\title{
Cohort Studies with Mortality Data from the Brazilian Population: a Rising National Requirement
}

\author{
Thiago Veiga Jardim \\ Universidade Federal de Goiás - Liga de Hipertensão Arterial, Goiânia, GO - Brazil \\ Short Editorial related to the article: Elevated High-Sensitivity Troponin I in the Stabilized Phase after an Acute Coronary Syndrome Predicts \\ All-Cause and Cardiovascular Mortality in a Highly Admixed Population: A 7-Year Cohort
}

Cohort studies assessing mortality predictors are extremely important to determine public health priorities and are a central issue in clinical decision making. These studies are particularly relevant in low-to-middle income countries whereas resources addressed to health care are limited and can be better managed. Nevertheless, traditionally epidemiological studies assessing the impact of different risk factors on mortality have historically been conducted in high-income countries. ${ }^{1}$

Results from cohort studies conducted in the developed world have been extrapolated and widely used in developing countries. This can be an issue given population and disease management differences, reinforcing the need to increase publications of long-term follow-up cohort studies with mortality data from low-to-middle income countries.

Efforts have been made along the years by a number of research groups in Brazil and around the world to progressively generate mortality data from low-to-middle income countries that are accurate, reliable and derived from well-designed cohort studies. In this number of ABC Cardiol, Castro et al. ${ }^{2}$ published one of the many examples of these efforts.

Aiming to assess the hypothesis that elevated levels of High-Sensitivity Troponin I (hs-cTnl) measured 25 to 90 days after an acute coronary syndrome (ACS) are associated with higher all-cause and cardiovascular mortality the authors used data from the ERICO study (Strategy of Registry of Acute Coronary Syndrome). ${ }^{3,4}$ This prospective cohort was designed to investigate the ACS epidemiology in Brazil and conducted at a secondary general hospital in São Paulo. Patients who were 35 years old or older with the acute coronary syndrome were enrolled consecutively. Sociodemographic, medical, and treatment data were obtained at admission, along with the blood sample collection. After 30 days, medical history was updated, blood and urinary samples were recollected, and additionally, a retinography, carotid intima-media thickness, heart rate variability and pulse-wave velocity were performed. Food frequency, physical activity, sleep apnea and depression were evaluated using specific questionnaires. Six months and annually after the acute event, telephone information was collected. ${ }^{3,4}$

\section{Keywords}

Cohort Studies; Mortality; Public Health; Clinical Decision Making; Risk factors; Acute Coronary Syndrome.

Mailing Address: Thiago Veiga Jardim •

Universidade Federal de Goiás - Programa de Pós-Graduação em Ciências da Saúde - Primeira Avenida, s/n, Setor Leste Universitário, Goiânia, GO - Brazil E-mail: thiagoloirin@hotmail.com

DOI: $10.5935 /$ abc. 20190038
Levels of hs-cTnl in 525 patients 25 to 90 days after admission for an ACS event were measured and mortality data were obtained in a seven years follow-up period. Patients in the highest tertile of hs-cTnl had a greater hazard ratio (HR) for all-cause mortality compared to the lowest tertile, after adjustment for age, sex, known cardiovascular risk factors, medication use, and demographic factors (HR: 3.84, 95\% Cl: 1.92-8.12). These findings persisted after further adjustment for estimated glomerular filtration rate $<60 \mathrm{ml} / \mathrm{min} / 1.73 \mathrm{~m}^{2}$ and left ventricular ejection fraction $<0.40$ (HR: 6.53, 95\% Cl: 2.12-20.14). Cardiovascular mortality was significantly higher in the highest tertile after adjustment for age and sex (HR: 5.65, 95\% Cl: 1.94-16.47) and both in the first (HR: 4.90, 95\% Cl: 1.35-17.82) and second models of multivariate adjustment (HR: 5.89, 95\% Cl: 1.08-32.27). The authors conclude that elevated levels of hs-cTnl in the stabilized phase after an ACS event carry long-term prognostic information that is independent of comorbidities, renal function and left ventricular ejection fraction.

Although the findings are not completely unique as the authors mention in the manuscript, they are unique for the Brazilian population (a highly admixed population). Additionally, some aspects of Castro`s study improve its strengths. It was performed at a community hospital, without a specific cardiologic team which is the reality of most hospitals in Brazil. Large sample size and long-term follow-up, probably representing one of the most important studies with prognostic data using biomarkers in patients with ACS in our country. Death records of all patients (either contacted or not during follow-up) were obtained, suggesting no bias in the mortality from all causes assessment.

The main limitation of Castro's et al. ${ }^{2}$ study is the single centre aspect. It was discussed in the manuscript, but some aspects of the results should be highlighted since they minimize this limitation. Most participants were males and had a high prevalence of hypertension. Those sociodemographic characteristics and cardiovascular comorbidities are similar to those of large international records, such as the registry Global Acute coronary events (GRACE)..$^{5}$ Another similarity with international trends ${ }^{6}$ is in the frequency of ST-segment elevation myocardial infarction (41.5\% of the participants), which represented the most frequent type of SCA in Castro`s study. Similarities were also found with the Brazilian Registry of acute coronary syndromes (BRACE), which included hospitals from all regions of Brazil. Medications used in the first follow-up visit were similar in comparison with the treatment received at the discharge of the participants in the BRACE registry: ${ }^{7}$ use of aspirin (83.6\% vs. $86.0 \%$, respectively), clopidogrel (53.0\% vs. 50.1\%), beta-blockers (64.2\% vs. $69.8 \%$ ), ACE inhibitors/angiotensin receptor blockers (68.3\% vs. $70.6 \%$ ) and statins (76.4\% vs. $82.7 \%$ ). 


\section{Short Editorial}

ABC Cardiol is the most important journal dedicated to publishing cardiovascular research generated in Brazil. Publish national cohort studies with mortality data,

\section{References}

1. Inuzuka S, Jardim PCV, Abrahams-Gessel S, Souza LG, Rezende AC, Perillo $\mathrm{NB}$, et al. Self-rated health status and illiteracy as death predictors in a Brazilian cohort. PLoS One. 2018;13(7):e020050

2. Castro LT, Santos IS, Goulart AC, Pereira AC, Staniak HL, Bittencourt MS, et al. Elevated High-Sensitivity Troponin I in the Stabilized Phase after an AcuteCoronary Syndrome Predicts All-Cause and Cardiovascular Mortality in a Highly Admixed Population: A 7-Year Cohort. Arq Bras Cardiol. 2019; 112(3):230-237.

3. Goulart A, Santos IS, Sitnik D, Staniak HL, Fedeli LM, Pastore CA, et al. Design and baseline characteristics of a coronary heart disease prospective cohort: two-year experience from the strategy of registry of acute coronary syndrome study (ERICO study). Clinics (Sao Paulo). 2013;68(3):431-4.

4. Santos IS, Goulart AC, Brandão RM, Santos RC, Bittencourt MS, Sitnik $D$, et al. One-year mortality after an acute coronary event and its clinical predictors: the ERICO study. Arq Bras Cardiol. 2015;105(1):53-64. particularly cardiovascular mortality data should be a priority. In this number, this priority was fulfilled with a great manuscript.
5. Goodman SG, Huang W, Yan AT, Budaj A, Kennelly BM, Gore JM, et al; Expanded Global Registry of Acute Coronary Events (GRACE2) Investigators. The expanded Global Registry of Acute Coronary Events: baseline characteristics, management practices, and hospital outcomes of patients with acute coronary syndromes. Am Heart J. 2009;158(2):193-201.

6. Roger VL, Weston SA, Gerber Y, Killian JM, Dunlay SM, Jaffe AS, et al. Trends in incidence, severity, and outcome of hospitalized myocardial infarction. Circulation. 2010;121(7):863-9.

7. Nicolau JC, Franken M, Lotufo PA, Carvalho AC, Neto JA, Lima FG, et al. Use of demonstrably effective therapies in the treatment of acute coronary syndromes: comparison between different Brazilian regions. Analysis of the Brazilian Registry on Acute Coronary Syndromes (BRACE). Arq Bras Cardiol. 2012;98(4):282-9. 